\title{
Programmed cell death without DNA fragmentation in the jimpy mouse: secreted factors can enhance survival
}

\author{
Pamela E. Knapp ${ }^{\star,}$, William P. Bartlett ${ }^{2}$, Laura A. Williams ${ }^{1}$, \\ Masahisa Yamada ${ }^{3}$, Kazuhiro Ikenaka ${ }^{3}$ and Robert P. Skoff ${ }^{4}$ \\ 1 Department of Anatomy and Neurobiology, University of Kentucky, Lexington, \\ Kentucky, USA \\ ${ }^{2}$ School of Physical Therapy, Texas Women's University, Houston, Texas, USA \\ ${ }^{3}$ National Institute for Physiological Science, Okazaki, Japan \\ ${ }^{4}$ Department of Anatomy and Cell Biology, Wayne State University, Detroit, \\ Michigan, USA \\ * corresponding author: Pamela E. Knapp, Ph.D., Department Anatomy \& \\ Neurobiology, MN224 Chandler Medical Center, University of Kentucky, \\ Lexington, Kentucky 40536-0084, USA. tel: 606-323-3517; \\ fax: 606-323-5946; e-mail: peknapp@pop.uky.edu
}

Received 15.3.98; revised 14.9.98; accepted 5.10.98

Edited by M. Noble

\begin{abstract}
Jimpy is one of many related mutations affecting the myelin proteolipid protein gene that causes severe hypomyelination in the central nervous system (CNS). Underlying the hypomyelination is a failure of oligodendrocytes (OLs) to differentiate, and the premature death of large numbers of OLs during the developmental period. Previous light and electron microscopic evidence suggested that jimpy OLs die in a manner consistent with programmed cell death. We have used TUNEL staining as a biochemical marker for apoptosis in conjunction with immunostaining for $\mathrm{OL}$ and myelin markers. At 13-14 days postnatal, a time when the number of dying OLs in jimpy CNS is increased more than five times normal, there are only modest increases $(70 \%$ in spinal cord; $20 \%$ in cerebral cortex) in TUNEL labeled cells in mutant CNS tissues. The results in vitro are similar, and only a small per cent of TUNEL labeled cells have the antigenic phenotype of OLs. The discrepancy between numbers of dying and TUNEL labeled cells suggests either that most jimpy OLs do not undergo programmed cell death or that the biochemical pathways leading to their death do not involve DNA fragmentation which is detected by the TUNEL method. We also present evidence that jimpy OLs show increased survival and enhanced differentiation when they are grown in vitro in medium conditioned by cells lines which express products of the proteolipid protein gene. Cell lines expressing proteolipid protein and the alternatively spliced DM20 protein have differential effects on cell numbers and production of myelinlike membranes.
\end{abstract}

Keywords: jimpy mouse; apoptosis; TUNEL; cell death; development; glial cells; myelin; DM20; PLP
Abbreviations: TUNEL, terminal deoxynucleotide transferase dUTP nick end-label; OL, oligodendrocyte; PLP, proteolipid protein; MBP, myelin basic protein; jp, jimpy; CNS, central nervous system; CM, conditioned medium; CAD, caspase activated DNase

\section{Introduction}

The jimpy (jp) mutation is one of many mutations in the gene coding for myelin proteolipid protein (PLP). Jimpy mice exhibit a constellation of abnormalities, of which the most conspicuous is an almost total lack of myelin in CNS. ${ }^{1-3}$ The mutation is sex linked, since the PLP gene is located on the Xchromosome, but carrier female mice also exhibit delayed myelination that is reversed by 3 months of age. ${ }^{4}$ Owing to the lack of myelin and resulting respiratory paralysis, male jp mice die between 3-4 weeks of age. Previous studies have shown that normal numbers of oligodendrocytes (OLs) are born in the jp brain, but jp OLs fail to fully differentiate and die in large numbers at the onset of myelination in different brain regions. ${ }^{5,6}$ Most or all of the phenotypic characteristics of the jp OL are maintained when the cells are put into an in vitro environment. $^{7,8}$ Cultured jp OLs begin to differentiate in normal numbers, but never develop extensive process networks or large myelin-like membrane sheets. Most cells die without accumulating substantial amounts of myelin proteins, just as occurs in the jp brain.

Although most jp OLs will die before fully differentiating both in the brain and in culture, their survival can be prolonged by manipulating the in vitro environment. Jimpy OLs grown in medium conditioned by normal astrocytes survive in large numbers and are capable of producing myelin-like membranes which contain near normal amounts of myelin basic protein (MBP) and galactocerebroside. ${ }^{7}$ Some jp OLs cultured in astrocyte conditioned medium synthesize a form of PLP that conforms to the predicted, mutant sequence and which can be detected immunohistochemically. ${ }^{8}$

Since determining that the survival of jp OLs can be prolonged we have begun to study the cascade of events leading to OL death and to determine which steps along that pathway are subject to modification by epigenetic factors. Pyknotic jp OLs have the morphology of apoptotic cells, including early polarization and condensation of nuclear chromatin. ${ }^{6}$ Very occasionally pyknotic jp OLs are seen attached to thin myelin sheaths which had begun to ensheath an axon. The phenotype of the dying jp OLs has prompted us and others to look for biochemical evidence of programmed cell death in jp brains and in cultured jp cells. The results of these studies have been variable, with evidence both in support of ${ }^{9,10}$ and against ${ }^{11,12}$ its occurrence in jp and related PLP disorders. In order to clarify this issue, we have examined frozen sections of optic nerves and cerebral cortex from jp and normal 
animals at $12-14$ days of age for evidence of terminal deoxynucleotide transferase dUTP nick end-label (TUNEL) staining. While TUNEL labeling was increased by up to $70 \%$ within brain tissues, this modest increase does not account for the more than tenfold increase in pyknotic OLs which has been described in jp brains. ${ }^{6}$ In addition, we have conducted in vitro studies on primary cultures of jp cerebral cortex using fluorescent TUNEL staining in conjunction with staining for OL specific markers. Although our jp cultures contain many TUNEL labeled cells, TUNEL staining is only rarely co-localized with OL markers. Thus, if jp OLs undergo apoptosis, they do so without exhibiting the characteristic DNA fragmentation detected by the TUNEL technique.

Cell lines producing PLP gene products have been reported to secrete factors which increase the differentiation and survival of normal OLs. ${ }^{13}$ For this reason we have also tested whether conditioned media prepared from cells stably transfected with PLP or DM20 cDNAs can normalize the phenotype of jp OLs. Three different types of conditioned media had differential effects on OL phenotype and behavior. Measurements of cell area show that medium conditioned by DM20 transfected cells significantly increased the size of myelin-like membrane expansions relative to control jp cells and jp cells grown in PLP cDNA or vector transfected cells. Conditioned medium from PLP transfected cells more greatly affected cell numbers. Densitometric evidence suggests that one of the mechanisms by which this phenotypic reversal may be accomplished involves an upregulation of mRNAs for myelin proteins.

\section{Results \\ Cell Death in vivo and in vitro}

TUNEL staining of $20 \mu \mathrm{m}$ frozen sections revealed only a small increase in numbers of labeled cells in any jp CNS region. TUNEL-labeled cells were quantified in three normal and three jp animals in three adjacent cross sections of lumbar level spinal cord and three adjacent mid-sagittal sections of cerebral cortex at 13-14 days of age. As shown in Table 1 , the mean number of TUNEL-labeled cells was increased by $70 \%$ in jp spinal cord and only $16 \%$ in jp cortex as compared to those same regions in normal CNS. Detection of TUNEL labeled cells was unambiguous in all sections. The staining resulted in minimal background color. In most instances TUNEL labeling was quite intense with an occasional lightly stained cell likely representing an earlier stage of apoptotic death (Figure 1). Overdevelopment of the sections did not result in an increase in numbers of TUNEL+ cells. There was no apparent staining of red blood cells within blood vessels, suggesting that background levels of peroxidase had been quenched and did not create a detection artifact.

The morphology of TUNEL stained cells in jp sections was not different from that in normal sections (Figure 1). Staining was contained in a more or less spherical region. Sometimes an intense periphery of staining surrounded a less well stained core. More often, there was peripheral and core staining of variable intensity. The location of the staining is largely nuclear. When surrounding cytoplasm can be identified it is obviously unstained.

TUNEL+ cells in both jp and normal cultures had a similar morphology and resembled TUNEL+ cells in tissue sections. Most cells exhibited a darkly stained, spherical region presumed to be the nucleus surrounded by a rounded region of unstained cytoplasm. Rarely, TUNEL+

Table 1 Numbers of TUNEL+cells in two CNS regions in 13-14 day normal and jimpy brains

\begin{tabular}{lrc}
\hline & Normal & Jimpy \\
\hline Spinal cord & $9(4.3)$ & $15(5.3)$ \\
Cerebral cortex & $83(9.0)$ & $96(9.1)$ \\
\hline
\end{tabular}

Numbers in parenthesis indicate standard errors of the mean $n=3$ for all samples
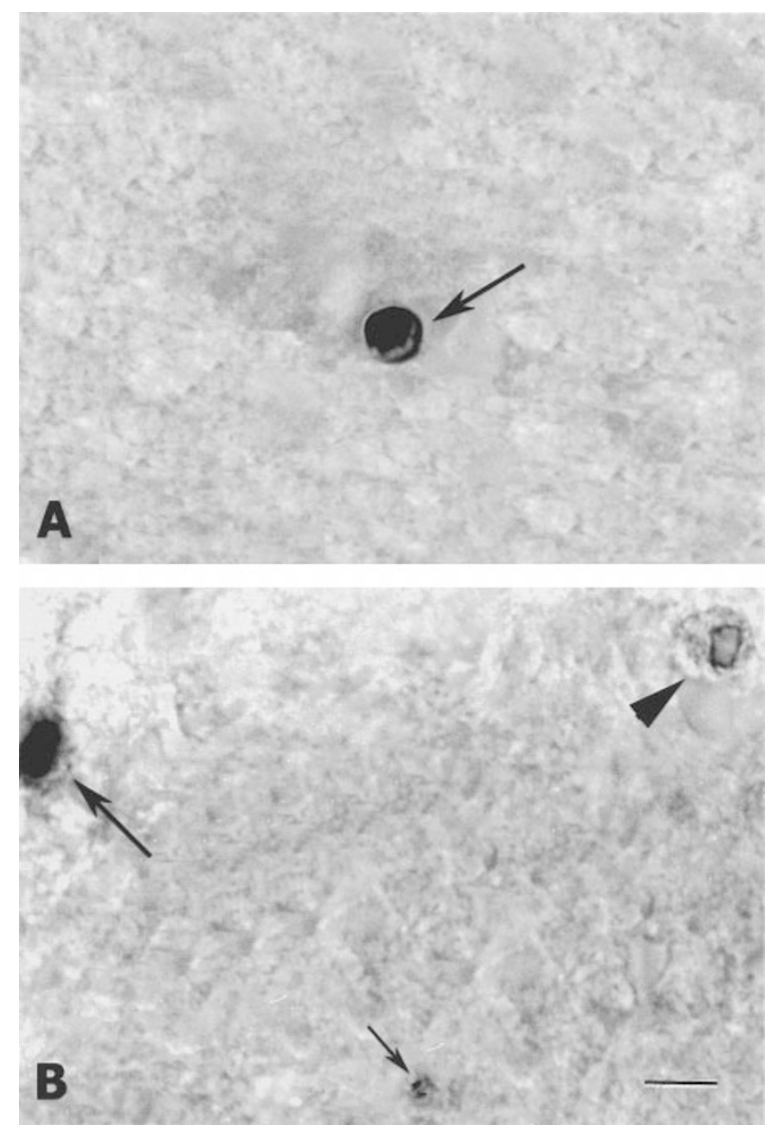

Figure 1 TUNEL-labeled cells in $20 \mu \mathrm{m}$ frozen sections of 12 day postnatal normal (A) and jimpy (B) spinal cord. Reaction product is largely confined to the nucleus, around which a spherical region of unstained cytoplasm can be detected. Cells exhibit a gradient of staining intensity. Most are quite dark (large arrows), although occasional cells show only slight color development at the nuclear periphery (arrowhead in B). Some labeled structures have an iregular shape or small size (small arrow in B) due to the angle of section through the cell. The jp section shown in $\mathbf{B}$ is misleading since it has three apoptotic cells and gives the impression that these cells are much more abundant than in normal spinal cord tissue. Cell counts show this is not the case. Bar $=10 \mu \mathrm{m}$ 
cells which were also immunostained for the $\mathrm{O}_{4}$ antigen were not rounded but had $\mathrm{O}_{4}$ staining in small cytoplasmic extensions (Figure 2). Staining within the nucleus was not necessarily uniform. Table 2 and Figure 2 show the results of combined TUNEL and $\mathrm{O}_{4}$ immunostaining to identify apoptotic OLs. Staining was done at 6 and 12 days of in vitro age since this is a time when OLs are lost in great
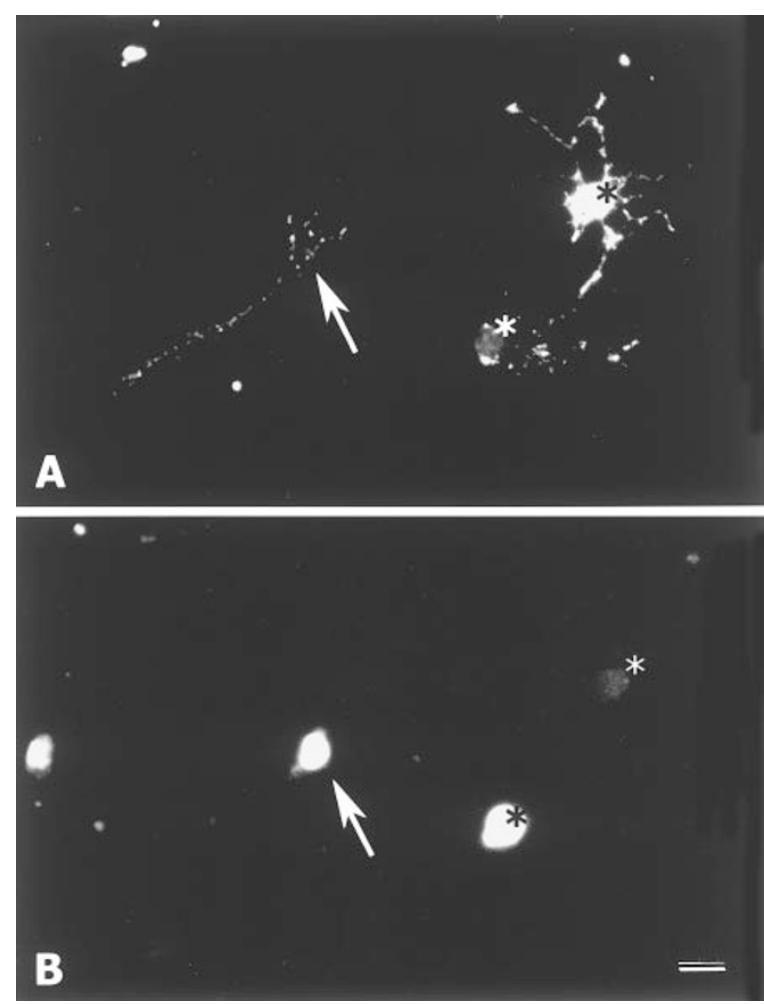

Figure 2 Double staining for TUNEL and $\mathrm{O}_{4}$ in a 12 day jimpy culture. (B) There are three cells whose nuclei are brightly fluorescent with TUNEL staining. (A) The TUNEL+ cell indicated by the arrow also shows punctate staining for the $\mathrm{O}_{4}$ antibody. Asterisks in $\mathbf{A}$ and $\mathbf{B}$ are positioned to indicate nearby cells as reference points. Bar $=20 \mu \mathrm{m}$ numbers from jp cultures, presumably due to cell death. ${ }^{7}$ At 6 days in vitro jp and normal cultures show similar numbers of TUNEL+ cells and double labeled TUNEL $+\mathrm{O}_{4}+$ cells. The percentage of $\mathrm{O}_{4}+$ cells that are TUNEL + is also similar $(<1.5 \%)$ and this does not change as cultures age. The small difference in TUNEL $+\mathrm{O}_{4}+$ cell number between normal and jp cultures at 12 days in vitro is not significant. Thus, even though OLs are being lost from jp cultures with increasing age, they apparently do not exhibit TUNEL staining.

\section{Effect of conditioned medium on mRNA production in jimpy OLs}

Conditioned medium (CM) from normal astrocytes has been shown to enhance the differentiation of jp OLs both by increasing levels of MBP and PLP and by enhancing membrane output. ${ }^{7,8}$ To determine whether these effects might be due to changes at the mRNA level we grew jp primary glial cultures in astrocyte CM for 14-16 days and then performed in situ hybridization and densitometric measurements to determined the relative expression of mRNAs for MBP and PLP. Growth in astrocyte CM had differential effects on MBP and PLP mRNAs. In situ hybridization for both MBP and PLP mRNA showed that the size of OL cell bodies in jp cultures was smaller than in normal cultures but that soma size was increased after growth in astrocyte CM (Figure 3). As judged by the optical density (OD) of in situ reaction product in individual cells, the level of PLP mRNA was increased in both normal and jp OLs grown in astrocyte CM (Figures 3 and 4). The effect of astrocyte CM was creater on normal OLs (165\% of control) than on jp OLs (129\% of control). Thus, both cell size and OD measurements indicated an increase level of PLP mRNA in treated jp OLs. In contrast, astrocyte $\mathrm{CM}$ had no effect on OD levels of MBP mRNA in either normal or jp OLs (Figures 3 and 4).

MBP mRNA is distributed both within the cell body and out into the processes of normal OLs, but was confined to the cell body region of jp OLs even though most jp OLs had a small network of processes. Growth in astrocyte CM

Table 2 Numbers of TUNEL ${ }^{+}$and $\mathrm{O}_{4}{ }^{+}$cells and the percentage of $\mathrm{O}_{4}{ }^{+}$cells with TUNEL labeling normal and jimpy cultures at two ages

\begin{tabular}{|c|c|c|c|c|c|}
\hline & TUNEL $^{+}$cells & $\mathrm{O}_{4}^{+}$cells & TUNEL $^{+} / \mathrm{O}_{4}{ }^{+}$ & $\% \mathrm{O}_{4}{ }^{+}$cells that are TUNEL $^{+}$ & \\
\hline \multicolumn{6}{|c|}{6 Day cultures } \\
\hline \multirow[t]{4}{*}{ Normal } & 202 & 987 & 8 & $0.8 \%$ & \\
\hline & 206 & 71 & 2 & $2.8 \%$ & Mean 1.4 \\
\hline & 150 & 73 & 1 & $1.4 \%$ & \\
\hline & 200 & 404 & 2.5 & $0.6 \%$ & \\
\hline \multirow[t]{4}{*}{ Jimpy } & 204 & 582 & 6 & $1.0 \%$ & \\
\hline & 174 & 229 & 1 & $0.4 \%$ & Mean 1.0 \\
\hline & 324 & 390 & 3.5 & $0.9 \%$ & \\
\hline & 195 & 194 & 3 & $1.5 \%$ & \\
\hline \multicolumn{6}{|c|}{12 Day cultures } \\
\hline \multirow[t]{2}{*}{ Normal } & 55 & 1056 & 2.5 & $0.24 \%$ & Mean 0.27 \\
\hline & 198 & 663 & 2 & $0.30 \%$ & \\
\hline \multirow[t]{3}{*}{ Jimpy } & 103 & 677 & 5 & $0.74 \%$ & \\
\hline & No data & 83 & 0.5 & $0.60 \%$ & Mean 0.86 \\
\hline & 173 & 200 & 2.5 & $1.25 \%$ & \\
\hline
\end{tabular}




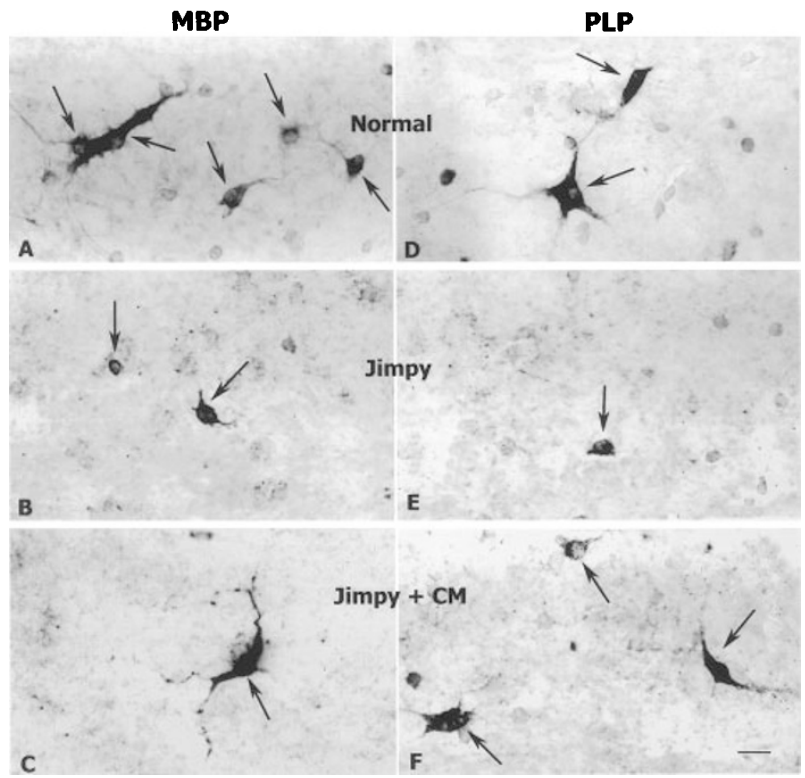

Figure 3 In situ hybridization using digoxigenin labeled CDNA probes for MBP $(\mathbf{A}-\mathbf{C})$ and PLP $(\mathbf{D}-\mathbf{F})$. (A and $\mathbf{D})$ show the distribution and density of MBP and PLP mRNAs, respectively, in normal OLs. (B and $\mathbf{E}$ ) show the distribution and density of MBP and PLP mRNAs, respectively, in untreated jp OLs. Note that the size of the jp cell soma is generally smaller than for normal OLs and that MBP mRNA is restricted to the region of the soma. ( $C$ and $\mathbf{F}$ ) show the distribution and density of MBP and PLP mRNAs, respectively, when jp OLs are grown in medium conditioned by normal astrocytes. Cell soma are increased in size in treated jp cultures, and MBP mRNA can now be detected in cell processes, reflecting the distribution typical of many normal OLs. Bar $=20 \mu \mathrm{m}$ promoted the redistribution of $\mathrm{MBP}$ mRNA into $\mathrm{OL}$ processes (Figure 3 ). Thus, although the average OD of MBP mRNA reaction product was unaffected, the increased area over which the mRNA was distributed suggests an increase in MBP mRNA content per cell. PLP mRNA is normally found within the region of the cell body and this localization was not changed by these growth conditions.

\section{Effect of conditioned medium on proliferation, survival and morphology of jimpy OLs}

A recent report by Nakao et al ${ }^{13}$ suggested that medium conditioned by cell lines transfected with PLP or DM20 cDNAs could influence the survival and development of normal OLs in vitro. We tested whether expression of the PLP gene affected the ability of $\mathrm{CM}$ to normalize the numbers or phenotype of jp OLs. Jp cells were grown for 14-16 days in medium conditioned by untransfected NIH3T3 cells or NIH3T3 cells transfected with $\mathrm{pDL}+$ vector alone or with PLP or DM20 cDNAs. Numbers of $\mathrm{O}_{1}$ and MBP stained cells in jp cultures were increased significantly by all types of CM (Figure 5). The PLP transfected cell line had the greatest effect on cell number, causing a 16-fold increase in $\mathrm{O}_{1}+$ cells (232 to 3876 , average of three experiments, data not shown) and a 21 -fold increase in MBP+ cells per coverslip (79 to 1659, average of three experiments, data not shown). Importantly, the increase in numbers of $\mathrm{O}_{1}+$ amd MBP+ cells in PLP-CM was significantly more than the increase seen in either DM20CM, NIH3T3-pDL CM or CM from untransfected NIH3T3 cells $(P<0.05)$. CM from the latter three lines all caused

\section{MBP Optical Density}

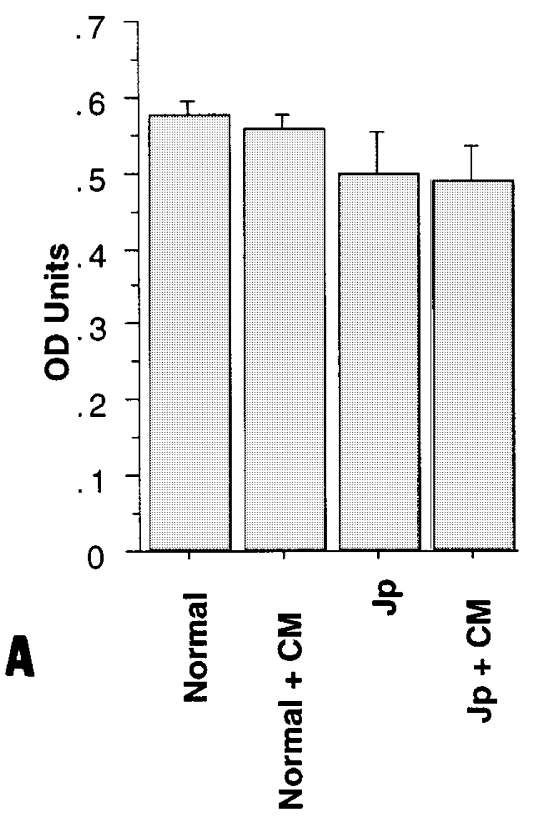

PLP Optical Density

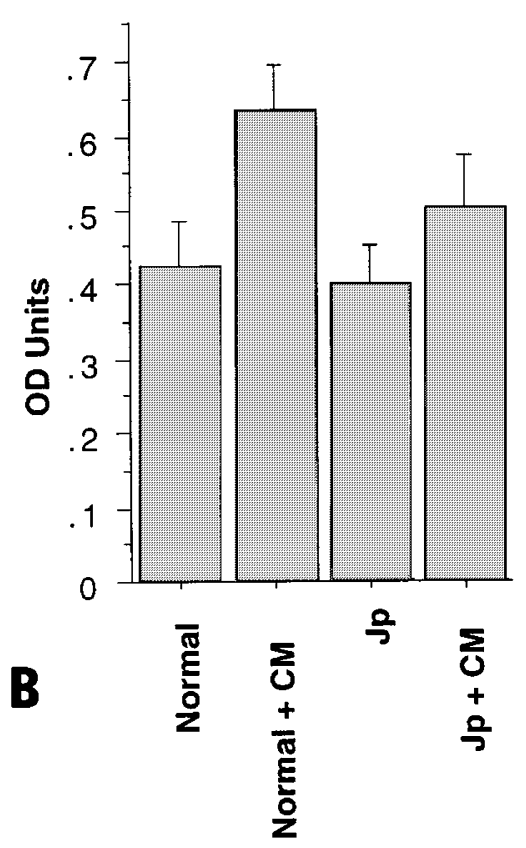

Figure 4 The effect of astrocyte conditioned medium (CM) on optical density (OD) of in situ hybridization reaction product for MBP and PLP mRNAs in normal and jp OLs. CM has no effect on MBP mRNA levels in either normal or jp OLs (A). The OD of PLP mRNA reaction product is increased in both normal and jp OLs after growth in astrocyte CM (B). $n=3$ for normal MBP; $n=6$ for jp MBP; $n=4$ for normal and jp PLP 
approximately tenfold increases in $\mathrm{O}_{1}+$ cells and MBP+ cells. Their effects were not significantly different from one another.

To determine whether numbers of jp OLs were increased due to changes in proliferation or survival we grew jp cultures in PLP-CM for 3, 7, 14 or 20 days before fixing and staining for $\mathrm{O}_{4}$ and $\mathrm{BrdU}$ or $\mathrm{O}_{4}$ and propidium iodide. We saw no reduction in numbers of $\mathrm{O}_{4}+\mathrm{OLs}$ with the fragmented or condensed nuclear morphology of apoptotic cells in the PLP-CM treated cultures at any time. This is not surprising since very few such cells were seen in control jp cultures $\left(0-1 \%\right.$ of total $\mathrm{O}_{4}+$ cells). Numbers of $\mathrm{O}_{4}+\mathrm{BrdU}+$ cells were significantly increased (by $50 \%$ ) after 7 days in PLP-CM, but not at any other time $(P<0.05)$. Cell numbers were analyzed using both Spearman's and Kendall's Rank Correlation non-parametric tests.

The percentage of jp OLs making myelin-like membrane sheets was unaffected by any of the NIH3T3 CM, remaining at approximately $15 \%$. However, media conditioned by the transfected cell lines had differential effects on the sizes of those myelin-like membranes. CM from the DM20 transfected line doubled the size of membranes produced by untreated jp OLs (Figures 5 and 6; $P<0.05$, unpaired $t$-test). Neither of the other two transfected lines affected membrane size.

\section{Discussion}

\section{Do jimpy oligodendrocytes undergo programmed cell death?}

When we first described the premature death of jp OLs we noted ultrastructural features consistent with programmed cell death, including polarization and condensation of nuclear chromatin prior to any disruption of the cytoplasm ${ }^{6}$ (Figure 7). The cells did not undergo lysis but rather were engulfed by phagocytic cells. The timing of their death also suggested a programmed event since OL death began at very prescribed
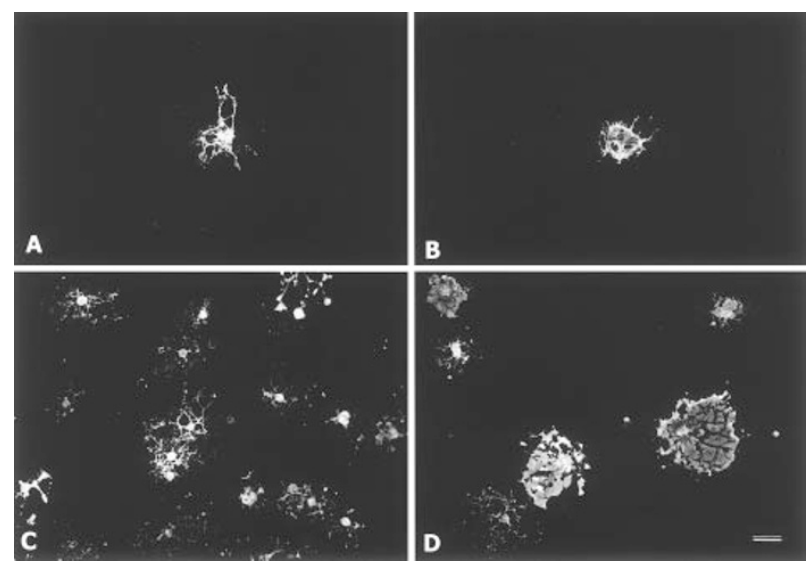

Figure $5 \quad O_{1}$ antibody staining to identify jp OLs on sister coverslips which were untreated (A and $\mathbf{B}$ ) or grown in conditioned medium from NIH3T3-PLP transfected cells $(\mathbf{C})$ or NIH3T3 DM20 transfected cells (D). Note that cell density is increased on both treated coverslips, but is highest on coverslips treated with NIH3T3-PLP conditioned medium (C). Average cell size is only affected by NIH3T3-DM20 conditioned medium (D). Bar $=25 \mu \mathrm{m}$

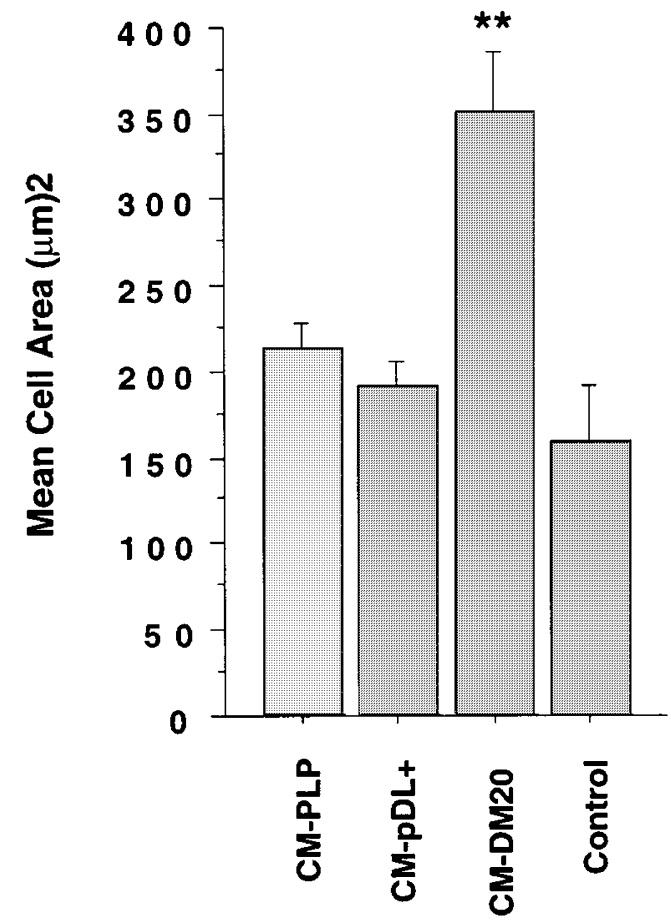

Figure 6 Effect of medium conditioned by $3 T 3$ cells transfected with pDL+ vector or PLP and DM20 cDNAs on OL cell and membrane size. **Different from control at $P<0.05$; different from CM-pDL+ and CM-PLP at $P<0.0001$. Different levels of significance are likely due to the smaller number of control jp OLs imaged

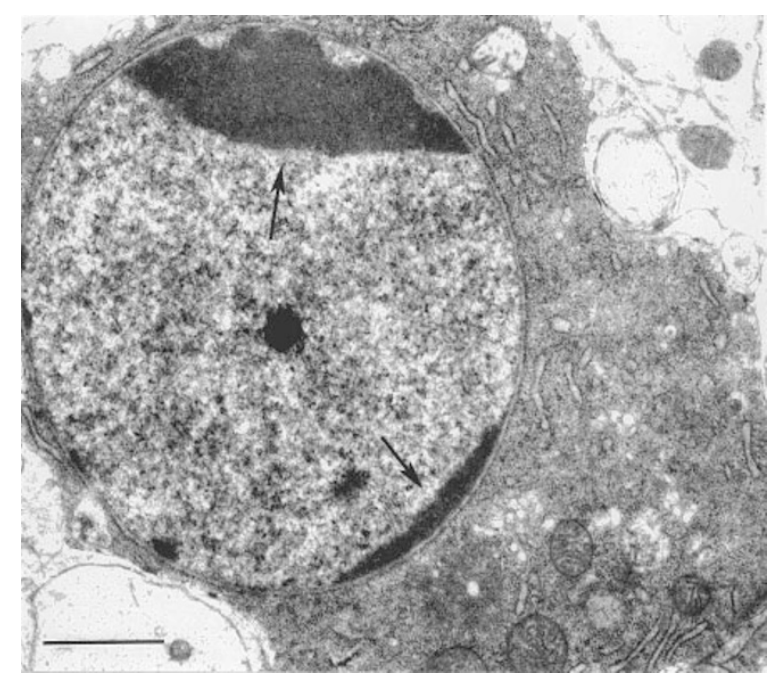

Figure 7 Electron micrograph of an OL from a 20 day jp mouse spinal cord which is still in the early stages of degeneration. The chromatin within the intact nuclear membrane has been polarized into two dark masses (arrows). The cytoplasm is still well ordered, although some mitochondria and strands of endoplasmic reticulum are beginning to show signs of swelling. The eccentric nucleus, stacks of endoplasmic reticulum, numerous free ribosomes and the relative density of cytoplasm and nucleoplasm are typical features of young OLs. $\operatorname{Bar}=1 \mu \mathrm{m}$ 
Table 3 Effect of astrocyte conditioned medium on production of PLP and MBP mRNAs in normal OLs and jimpy OLs

\begin{tabular}{|c|c|c|c|c|c|c|}
\hline & \multicolumn{3}{|c|}{ Normal OLs-MBP mRNA (OD) } & \multicolumn{3}{|c|}{ Jp OLs-MBP mRNA (OD) } \\
\hline & Control & $+\mathrm{CM}$ & $+\mathrm{CM} /$ control & Control & $+\mathrm{CM}$ & $+\mathrm{CM} /$ control \\
\hline Expt. 1 & 0.592 & 0.540 & $91 \%$ & 0.374 & 0.363 & $97 \%$ \\
\hline Expt. 4 & No data & & & 0.481 & 0.564 & $117 \%$ \\
\hline Expt. 5 & No data & & & 0.602 & 0.614 & $102 \%$ \\
\hline Expt. 6 & 0.539 & 0.527 & $98 \%$ & 0.321 & 0.350 & $109 \%$ \\
\hline Expt. 7 & 0.595 & 0.600 & $101 \%$ & 0.666 & 0.545 & $82 \%$ \\
\hline \multirow[t]{4}{*}{ Expt. 8} & No data & & & 0.551 & 0.511 & $93 \%$ \\
\hline & & Mean & $97 \%$ of control & & Mean & $100 \%$ of control \\
\hline & \multicolumn{3}{|c|}{ Normal OLs-PLP mRNA (OD) } & \multicolumn{3}{|c|}{ Jp OLs-PLP mRNA (OD) } \\
\hline & Control & $+\mathrm{CM}$ & $+\mathrm{CM} /$ control & Control & $+\mathrm{CM}$ & $+\mathrm{CM} /$ control \\
\hline Expt. 1 & 0.286 & 0.790 & $276 \%$ & 0.353 & 0.623 & $176 \%$ \\
\hline Expt. 2 & 0.358 & 0.535 & $150 \%$ & No data & & \\
\hline Expt. 3 & 0.474 & 0.553 & $117 \%$ & 0.402 & 0.499 & $124 \%$ \\
\hline Expt. 4 & No data & & & 0.306 & 0.322 & $105 \%$ \\
\hline \multirow[t]{2}{*}{ Expt. 7} & 0.578 & 0.669 & $116 \%$ & 0.542 & 0.590 & $109 \%$ \\
\hline & & Mean & $165 \%$ of control & & Mean & $129 \%$ of control \\
\hline
\end{tabular}

times corresponding with the onset of myelination in individual brain regions. By light microscopic criteria we estimated that up to $20 \%$ of the total OL population might by pyknotic at any one time. To further explore mechanisms of OL death in the jp CNS we have tried to determine: (a) whether the premature and seemingly coordinated loss of OLs occurs by programmed cell death; and (b) whether survival and differentiation of jp OLs can be enhanced by epigenetic factors related to PLP gene expression.

Tissues from jp CNS both in situ and in vitro were analyzed for the presence of apoptotic cells using the classic TUNEL method to end-label internucleosomal DNA fragments. Since many more glia were dying in jp than in normal spinal cord at both 11 days $(4.8 \times)$ and 16 days $(9.3 \times)$ of age, we expected quite a large increase in TUNEL+ cells in jp tissues at the age examined. Surprisingly, numbers of TUNEL+ cells were increased only very modestly in frozen sections of jp spinal cord (by $70 \%$ ) and cerebral cortex (by 16\%) (Table 1). These results were similar to those found in vitro, where less than a $20 \%$ increase in total TUNEL+ cells was seen in primary cultures of jp CNS at 6 and 12 days in vitro (Table 2). The in vitro studies combined TUNEL labeling with $\mathrm{O}_{4}$ immunohistochemistry to identify apoptotic cells that had antigenic characteristics of OLs. By two criteria there were few of these cells. Firstly, a very low percentage of the TUNEL+ cells expressed the OL marker (Table 2). Second, less than $1.5 \%$ of $\mathrm{O}_{4}+$ cells in either jp or normal cultures were TUNEL+.

Our results are in agreement with another study where TUNEL staining was used to investigate mechanisms of OL death in the jp CNS. ${ }^{11}$ In that study, numbers of TUNEL+ OLs were increased from 1.5-2 times in jp spinal cord and were within $\pm 25 \%$ of normal levels in sagittal brain sections at two stages. The dying cells had morphology similar to that shown in the present report although completely different fixation and TUNEL labeling systems were used. Williams and Gard $^{12}$ reported no increase in TUNEL labeled jp cells in a preliminary report. More recently, Gow et al $^{9}$ studied TUNEL labeling in the myelin synthesis deficient mouse, another PLP mutant, and showed a 2-3-fold increase over normal at 10 days postnatal in various regions of spinal cord. Surprisingly, the absolute numbers of dying cells in that study were tenfold lower than in our original study of cell death in jp. ${ }^{6}$ While some of that difference must be due to morphological versus TUNEL identification of the dying cells, the difference is striking when one considers that terminal deoxytransferase labeling usually detects more apoptotic cells than simple morphological identification. ${ }^{14}$ Although msd and jp are both PLP mutants, the differences in the two mutations may preclude any direct comparison of numbers of dying cells. Since the present results and others cited above all show some increased TUNEL labeling or a faint $180 \mathrm{bp}$ DNA ladder, ${ }^{9}$ it is clear that some increased DNA fragmentation can be documented in both jp and msd CNS tissues. However, it is also clear that many dying OLs either do not undergo DNA fragmentation or are phagocytized prior to the point at which it can be biochemically detected.

Our findings raise the perplexing question of how morphologic findings and TUNEL labeling can lead to such different conclusions. In situ visualization with terminal deoxytransferase usually detects many more apoptotic cells than can be identified using morphologic techniques. ${ }^{14}$ However, if TUNEL labeling reliably detects apoptosis in our cells, then the much smaller than expected numbers of TUNEL+ cells suggest that apoptosis is not the main path towards OL death within the jp brain. Several more variables must be considered in the in vitro setting. Since the majority of cells in primary cultures are astrocytes, the total number of TUNEL+ cells in jp cultures may not be a good index of the number of dying OLs. However, the number of $\mathrm{O}_{4}+$ TUNEL + cells is expected to be increased, especially since $\mathrm{O}_{4}+$ ]TUNEL+ cells will not be phagocytized in vitro. One explanation for the shortage of $\mathrm{O}_{4}+\mathrm{TUNEL}+$ cells could be that many dying OLs lose $\mathrm{O}_{4}$ antigenicity. This seems unlikely, since the majority of jp OLs are $\mathrm{O}_{4}+$ 
and remain so until they die. Even the membranous remnants of dead OLs which adhere to the culture surface stain positively for $\mathrm{O}_{4}$. It also seems peculiar that we rarely find TUNEL+cells in culture or in the brain which have a process-bearing morphology. Most TUNEL+cells are rounded in both situations. In contrast, the cytoplasm and processes of dying OLs identified by their abnormal chromatin in vivo remains intact until degeneration is quite advanced (Figure 7). ${ }^{6}$ And preliminary in vitro observations show that even dying jp OLs with intracellular $\mathrm{Ca}^{2+}$ levels elevated above $1 \mu \mathrm{M}$ retain a process-bearing morphology (Knapp and Hauser, unpublished results). The above findings suggest that TUNEL labeling does not reliably detect jp OLs in early stages of death in situ or in culture.

Although TUNEL labeling and DNA laddering have been used as definitive measures of programmed cell death, evidence is emerging that programmed cell death may occur in the absence of these events. Both techniques detect the internucleosomal cleavage of DNA into $180 \mathrm{bp}$ fragments. However, there are numerous documented instances in which programmed cell death occurs in the absence of internucleosomal DNA cleavage. ${ }^{15-19} \mathrm{~A}$ further confounding factor is that the temporal relationship between nuclear condensation and DNA fragmentation may not be constant since a recent study shows that nuclear changes can occur as a much earlier event than DNA fragmentation. ${ }^{20}$ The recent identification of a caspase activated DNase (CAD) presumed to be responsible for DNA degradation into $180 \mathrm{bp}$ fragments provides insight as to how apoptosis might occur in the absence of TUNEL labeling. $^{21,22}$ Overexpression of the CAD inhibitor ICAD completely blocks DNA fragmentation as determined by laddering on gels, but does not prevent cell death assayed using annexin-V or MTT. ${ }^{22}$ This suggests that death can occur via cleavage of vital cellular substrates, presumably by activated caspases, without the DNA fragmentation detected by TUNEL labeling.

Our morphologic studies have clearly shown that nuclear changes are a characteristic feature of OLs within jp CNS and have documented a progression of nuclear and cytoplasmic changes leading to pyknotic cell bodies. ${ }^{6}$ The cells dying in the brain and in vitro bear no resemblance to necrotic cells. We believe that jp OLs are undergoing apoptosis or programmed cell death, but that the signaling pathway which leads to jp OL death may not necessarily involve the DNA fragmentation detected by TUNEL staining. Even if DNA fragmentation were delayed, TUNEL labeling should readily detect DNA fragments in the cultures since dying cells are not phagocytized.

\section{Jimpy oligodendrocyte survival and differentiation can be enhanced}

Although most jp OLs die before they can produce myelin in situ, a small percentage of axons within jp CNS are myelineated, albeit thinly and in the absence of PLP. ${ }^{1,23} \mathrm{~A}$ parallel situation occurs in culture where most jp OLs will die before fully differentiating although a few cells maintain small membranous sheets which contain myelin proteins. ${ }^{7,8}$ Since some jp OLs survive for reasonably long periods and exhibit myelinating behavior, these cells may not be destined to die prematurely but may be induced to do so by some combination of internal (genetic) and external (epigenetic) factors. This idea was supported by the finding that factors present in medium conditioned by normal astrocytes could increase the survival and differentiation of jp OLs. ${ }^{7}$ When grown in astrocyte CM, jp OLs produce large, myelin-like membranes which immunostain as intensely for myelin glycolipids and MBP as membranes in normal OLs. ${ }^{7,8}$ Some jp OLs are even found to immunostain for the predicted mutant form of PLP. ${ }^{8} A$ recent report that jp OLs were not influenced by astrocyte $\mathrm{CM}$ is likely due to significant differences in both culture and CM preparation. ${ }^{24}$

In a similar vein, CM from cell lines transfected with PLP or DM20 cDNAs is reported to influence the development of normal OLs. ${ }^{13}$ We examined whether expression of PLP/ DM20 might play a role in the ability of a cell line to rescue the phenotype of jp OLs. NIH3T3 cells, which do not normally express PLP or DM20, were stably transfected with PLP or DM20 cDNAs as previously reported. ${ }^{13}$ All cell lines, including untransfected NIH3T3 cells and cells transfected with pDL+ retroviral vector alone, secreted factors which increased numbers of jp OLs. Importantly, both the PLP and DM20 transfected lines had effects above and beyond that of the control cell lines. And the effects of the PLP and DM20 expressing lines were different. CM from the PLP transfected line doubled the numbers of $\mathrm{MBP}+$ and $\mathrm{O}_{1}+$ jp OLs seen in cultures treated with any other CM. BrdU studies show that at least a part of this effect is due to increased proliferation. CM from the DM20 transfected line was the only $\mathrm{CM}$ which significantly affected the size of jp myelin-like membranes (Figures 5 and 6). Thus it appears that NIH3T3 cells by themselves produce factors which promote increased numbers of jp OLs, but that expression of PLP or DM20 confers additional abilities to rescue their phenotype. The effects of the NIH3T3 conditioned media on the phenotype of jp OLs are somewhat different from those of normal astrocyte $\mathrm{CM}$ which had been shown to increase both OL numbers and the percentage of OLs which produce myelin-like membranes. $^{7,8}$ None of the NIH3T3 lines affected the percentage of OLs which produce membrane sheets. This percentage remained at $10-15 \%$.

Our results suggest that secreted factors may affect jp OL phenotype by regulating the cellular content of critical mRNAs either by upregulating transcription or by increasing mRNA stability. In addition to increasing the size of the cell soma, growth in astrocyte CM increased the optical density of in situ hybridization reaction product for PLP mRNA in both normal and jp OLs (Figures 3 and 4). Since PLP mRNA is found throughout the entire cell soma, the total cell content of PLP mRNA must be increased. MBP mRNA content is increased by virtue of the increased normal size and a wider area of distribution. Secreted factors appear to regulate distribution of $\mathrm{mRNA}$ since MBP mRNA moves into OL processes in treated jp cells but is retained in the cell soma in controls (Figure 3). Distribution into processes more closely parallels the situation in normal OLs. Since restriction of MBP mRNA to the cell soma has been correlated with physical content between astrocytes and 
normal OLs, ${ }^{25}$ it is interesting that the effect of astrocyte secreted factors has an opposite effect on many jp OLs.

In earlier experiments primary fibroblast $\mathrm{CM}$ did not increase jp OL survival or differentiation. This suggests that specific factor(s) secreted by certain cell types can provide tropic support not available to jp OLs either in situ or from the bedlayer of cells in primary jp cultures. Variable amounts or types and combinations of such factors could account for the distinct effects of CM from different sources. One intriguing possibility raised by the differential effects of NIH3T3 cell lines transfected with PLP or DM20 cDNAs is that the PLP gene itself may endow normal OLs with an 'autotrophic' ability. If normal activity of the PLP gene initiates production of factors which support or are requisite for OL survival and differentiation, this support might be lost with particular PLP mutations. Trophic factors could be supplied by other cells within microenvironments in the mutant CNS, or by replacement conditions in vitro. This theory fits our in vitro observations and would explain both the increased differentiation of jp OLs in heterotypic transplants ${ }^{26}$ and the survival and partial differentiation of small numbers of jp OLs in situ. However, studies on transgenic PLP and DM20 knockouts ${ }^{27,28}$ indicate that there must also be a dominant-negative consequence of the jp mutation.

Our present evidence substantiates that numbers of jp OLs can be normalized and that their differentiation can be greatly enhanced when the cells are removed from the brain and grown in vitro under certain conditions. The findings suggest that these phenotypic changes may be accomplished by a variety of mechanisms including enhanced proliferation and upregulation of myelin protein mRNAs.

\section{Materials and Methods}

\section{Cell culture}

Primary cultures of mixed glial cells were prepared from 2 day postnatal mouse brains using our published procedures. ${ }^{29}$ Briefly, cerebral cortices were removed and single cell suspensions obtained by enzymatic and mechanical disruption, followed by filtration through a $35 \mu \mathrm{m}$ pore nylon mesh (Tetko, East Elmsford NY, USA). $2.5 \times 10^{5}$ cells were plated onto poly-L-lysine coated glass coverslips $(12 \mathrm{~mm}$ diameter; Bellco Glass, Vineland, NJ, USA), and grown in Dulbecco's Modified Eagle's Medium containing 10\% fetal calf serum (Hyclone Labs, Logan, UT, USA), $6 \mathrm{mg} \%$ glucose, $\mathrm{NaHCO}_{3}$ and antibiotics (10\% FCS medium). Medium was changed on the day after plating and at 3 day intervals. Cultures were prepared from both jp and normal animals in exactly the same manner. To confirm that suspected jp animals carried the gene, cervical spinal cords were removed, embedded in plastic, sectioned at $2 \mu \mathrm{m}$ and examined for the presence of incipient myelin wrappings within the ventral funiculus. By 2 days postnatal myelination has begun in this region of the normal spinal cord, but it is completely lacking in spinal cords from jp animals. ${ }^{7}$

\section{Immunohistochemistry and TUNEL labeling}

Cultured OLs were identified by immunostaining with either the $\mathrm{O}_{1}$ or $\mathrm{O}_{4}$ antibody. Both are $\mathrm{IgM}$ rat monoclonal antibodies to glycolipids which specifically identify OLs in CNS tissues. ${ }^{30} \mathrm{O}_{1}$ is specific for galactocerebroside while $\mathrm{O}_{4}$ recognizes sulfatide, the sulfated form of galactocerebroside. ${ }^{31}$ Staining for $\mathrm{O}_{1}$ and $\mathrm{O}_{4}$ is done on live cells at $4{ }^{\circ} \mathrm{C}$ with primary antibody applied for $20 \mathrm{~min}$, followed by visualization with goat-anti-mouse-IgM coupled to either -RITC or -FITC. $\mathrm{O}_{4}$ immunostaining was combined with a fluorescent TUNEL labeling procedure. At $5-6$ days and at 12 days live cells were stained with $\mathrm{O}_{4}$ antibody, then fixed in $4 \%$ paraformaldehyde for $10 \mathrm{~min}$. The cells were incubated in $0.5 \%$ saponin in phosphate buffered saline for 20 min. A TUNEL in situ cell death kit was used to detect apoptosis following the directions for fluorescent staining. Coverslips were than mounted using Prolong anti-fade mounting medium (Molecular Probes, Eugene, OR, USA). Some cell counts were done on preparations fixed and stained for MBP using a primary mouse-antiMBP (Sternberger Monoclonals, Baltimore, MD, USA; $1: 200,1 \mathrm{~h}$ ) and appropriate second antibody.

TUNEL staining was also performed on frozen sections of spinal cord and cerebral cortex taken from 13-14 day jp and normal pups. Animals were anesthetized with $9 \%$ chloral hydrate and transcardially perfused with $4 \%$ paraformaldehyde. Tissues were removed and left in fixative for $48 \mathrm{~h}$, then cryoprotected in a $25 \%$ sucrose solution. Tissue was embedded in OCT compound and $20 \mu \mathrm{m}$ frozen sections were cut and mounted onto Vectabond (Vector Laboratories, Burlingame, CA, USA) coated slides, then labeled using a TUNEL in situ cell death kit with 3-3' diaminobenzidine color development. Hydrogen peroxide was used to quench endogenous peroxidase activity. Cells were counted and quantified in each of three normal and jp animals in three adjacent cross sections of lumbar level spinal cord and three adjacent mid-sagittal sections of cortex. Numbers of TUNEL labeled cells in the three sample sections were averaged to calculate mean cell numbers in each region for each animal. All antibodies, kits and staining reagents were from Boehringer-Mannheim (Indianapolis, IN, USA) unless noted.

\section{Conditioned media experiments}

Several types of CM were produced for different experiments. We have previously shown that $\mathrm{CM}$ from primary astrocyte cultures increased the numbers of OLs in jp cultures as well as the size of their myelin-like membrane sheets and content of myelin proteins. ${ }^{7,8}$ To investigate the effects of astrocyte CM on myelin-specific mRNA production, medium was removed from confluent primary astrocyte cultures after $48 \mathrm{~h}$, supplemented with fresh medium (1:5), sterile filtered and placed onto normal or jp primary cultures 1 day after plating. Control coverslips were maintained in unconditioned $10 \%$ FCS medium. Astrocyte CM was changed every $48 \mathrm{~h}$ and treated coverslips were fixed and processed for in situ hybridization after 14-16 days.

For experiments testing the effects of other types of $\mathrm{CM}$ on jp survival and OL differentiation we used clonal lines of NIH3T3 cells which had been stably transfected with cDNAs for PLP or DM20. The PLP or DM20cDNAs were digested and cloned into the BamHI site of the $\mathrm{pDL}+$ retroviral vector and the resulting plasmid was introduced into the Psi2 packaging cell line by standard $\mathrm{CaPO}_{4}$ coprecipitation methods to obtain retroviral producer lines. ${ }^{13}$ Supernatants of transformed Psi2 cells were used to transduce NIH3T3 cells, which were then selected by G418 resistance. Control medium for the transfects was obtained from an NIH3T3 cell line transfected with the $\mathrm{pDL}+$ retroviral vector alone. CM was collected from clonal lines which constitutively expressed PLP or DM20 proteins as detected by immunohistochemistry. These cell lines were grown to confluency in serum-free medium. Supernatant was collected and concentrated to $10 \times$, then dialyzed against $5 \mathrm{mM}$ sodium phosphate buffer containing $50 \mathrm{mM} \mathrm{NaCl}$ (ph 7.4). The 
dialyzed concentrate was stored at $-70^{\circ} \mathrm{C}$. $30 \mu \mathrm{g} / \mathrm{ml}$ of concentrated supernatant was applied to primary jp cultures in $10 \%$ FCS medium on the day after plating. Control coverslips were maintained in unconditioned $10 \%$ FCS medium. Medium was replaced every other day and cells were fixed and processed for immunostaining and image analysis after 14-16 days.

\section{In situ hybridization}

Primary jp and normal cultures were fixed with $4 \%$ paraformaldehyde and dehydrated in $70 \%$ ethanol at $-20^{\circ} \mathrm{C}(10 \mathrm{~min})$. The cells were prehybridized at $55^{\circ} \mathrm{C}(10 \mathrm{~min})$ before application of linearized full length cDNA probes for MBP or PLP (Dr. Anthony Campagnoni, UCLA) which had been random primed with digoxigenin (Genius kit, Boehringer-Mannheim). Hybridization was for $18 \mathrm{~h}$ at $45^{\circ} \mathrm{C}$. After stringent washing $\left(4 \times-0.5 \times \mathrm{SSC}\right.$ with formamide; $\left.37^{\circ} \mathrm{C}\right)$ and treatment with $\mathrm{S} 1$ nuclease $\left(15 \mathrm{~min}, 37^{\circ} \mathrm{C}\right)$, an anti digoxigeninalkaline phosphatase conjugate was applied and color was developed using NBT-BCIP. Color development times were equal for control and experimental coverslips.

Optical density of MBP or PLP reaction products in individual OLs was measured using M1 Image Analysis Software (Imaging Research Corporation, St. Catherine's, Ontario, Canada). Because of variations in background color development across the coverslip, a background reading was taken next to each individual cell and then subtracted to compute the optical density for that cell. Fifty cells in each of 3-6 separate cultures were analyzed for all conditions (PLP and MBP mRNA levels in normal and jp cells, grown in CM or in $10 \% \mathrm{FCS}$ ). Random fields were selected and all positive cells within a field were quantified. To avoid the problem of variation in color development between individual experiments, mean optical densities were calculated and then expressed as a percentage of control value for that experiment.

\section{Cell area analysis}

After 12-13 days in CM from either PLP, DM20 or pDL+ transfected NIH3T3 cell lines, coverslips containing jp cells were immunostained using the $\mathrm{O}_{1}$ antibody and photographs were taken of random areas of the coverslip. Photographs were also taken of control jp cultures grown in unsupplemented 10\% FCS medium. To analyze the area of OLs grown under these four conditions, photographic negatives were scanned (Nikon Coolscan LS1000) and digitized. The areas of all individual cells that could be identified on the negatives were measured using Bioquant image analysis software $(R \& M$ Biometrics, Nashville, TN, USA). The same number of negatives, representing the same total coverslip area, was analyzed for each of the four media. Because of the effects of all three conditioned media on OL numbers, approximately 8-10 times more cells (183-223) were analyzed for each of those growth conditions than for the untreated jp OLs. Coverslips treated with conditioned medium from PLP transfected cells had such a high density of OLs that many cells overlapped and their boundaries could not be distinguished clearly enough for imaging. The number of cells imaged for this treatment group underestimates the total number of cells within the photographed regions. Mean cell areas for each group were calculated and significance was analyzed using an unpaired twotailed $t$-test (StatView, Abacus Concepts, Berkeley, CA, USA).

\section{Proliferation and survival studies}

In experiments designed to quantify proliferation we pulsed cells with $\operatorname{BrdU}(20 \mu \mathrm{M})$ at $37^{\circ} \mathrm{C}$ for $18 \mathrm{~h}$, then fixed and immunostained them using a monoclonal $\lg _{1}$ anti-BrdU antibody according to our published procedures (Knapp, 1992) ${ }^{32}$. BrdU was added directly to either control medium or PLP-CM at 4, 7, 14 or 20 days of growth. $\mathrm{O}_{4}$ staining was performed before $\mathrm{BrdU}$ staining in double-labeling procedures, using appropriate FITC and RITC labeled second antibodies. Two hundred random $\mathrm{O}_{4}+$ cells were examined for the presence of BrdU nuclear staining and duplicate coverslips were counted for each condition. As cultures aged the number of $\mathrm{O}_{4}+$ cells in the jp cultures diminished. In cases where there were less than 200 total $\mathrm{O}_{4}+$ cells on a coverslip we counted the total number.

To assess whether treatment with PLP-CM reduced the number of apoptotic OLs in the culture, we stained cells with propidium iodide at $4,7,14$ or 20 days in order to visualize nuclear morphology. $\mathrm{O}_{4}$ stained coverslips were treated with $1 \mathrm{M} \mathrm{HCl}$ to denature DNA, neutralized in sodium-phosphate buffer, $\mathrm{pH} 8.5$, then stained with $2 \mathrm{mg} \%$ propodium iodide for $1 \mathrm{~min}$. The nuclei of 200 random $\mathrm{O}_{4}+$ cells were examined for typical characteristics of apoptosis, including chromatin condensation and/or fragmentation. As in the proliferation studies, on coverslips with less than 200 total $\mathrm{O}_{4}+$ cells, all cells were examined. Both the proliferation and survival date were all analyzed using the paired $t$-test.

\section{Acknowledgements}

This work was supported by grants from the National Multiple Sclerosis Society (PEK; WPB; RPS); the National Center for Nervous, Mental and Muscular Disorders of the Ministry of Health and Welfare or Japan (KI); and the Nissan Science Foundation (KI).

\section{References}

1. Koeppen AH, Barron KD, Csiza CK and Greenfield EA (1988) Comparative immunocytochemistry of Pelizaeus-Merzbacher disease, the jimpy mouse, and the myelin-deficient rat. J. Neurol. Sci. 84: 315-327

2. Sidman RL, Dickie MM and Appel SH (1964) Mutant mice (Quaking and Jimpy) with deficient myelination in the central nervous system. Science 144: 309-311

3. Skoff RP and Knapp PE (1992) Phenotypic expression of X-linked genetic defects affecting myelination. In 'Myelin: Biology and Chemistry' Martenson $R$ ed. CRC Press, Boca Raton pp. 653-676

4. Bartlett WP and Skoff RP (1986) Expression of the jimpy gene in spinal cords of heterozygous female mice. I. An early myelin deficitfollowed by compensation. J. Neurosci. 6: 2802-2812

5. Ghandour MS and Skoff RP (1988) Expression of galactocerebroside in developing normal and jimpy oligodendrocytes in situ. J. Neurocytol. 17: 485498

6. Knapp PE, Skoff RP and Redstone DW (1986) Oligodendroglial cell death in jimpy mice: An explanation for the myelin deficit. J. Neurosci. 6: 2813-2822

7. Bartlett WP, Knapp PE and Skoff RP (1988) Glial conditioned medium enables jimpy oligodendroglia to express properties of normal oligodendrocytes: Production of myelin antigens and membranes. Glia 1: 253-259

8. Knapp PE, Skoff RP and Benjamins JA (1996) Epigenetic factors upregulate expression of myelin proteins in the dysmyelinating jimpy mutant mouse. J. Neurobiol. 29: 138-150

9. Gow A, Southwood CM and Lazzarini RA (1998) Disrupted proteolipid protein trafficking in oligodendrocyte apoptosis in an animal model of PelizaeusMerzbacher disease. J. Cell Biol. 140: 925-934

10. Vela JM, Dalmau I, Gonzalez B and Castellano B (1996) The microglial reaction in spinal cords of jimpy mice is related to apoptotic oligodendrocytes. Brain Res. 712: $134-142$

11. Skoff RP (1995) Programmed cell death in the dysmyelinating mutants. Brain Pathol. 5: 283-288

12. Williams II WC and Gard AL (1995) DNA fragmentation is not a prominent feature of oligodendrocyte death in the jimpy mutant. J. Neurochem. 64: S98C.

13. Nakao J, Yamada M, Kagawa T, Kim SU, Miyao Y, Shimizu K, Mikoshiba K and Ikenaka K (1995) Expression of proteolipid protein gene is directly associated with secretion of a factor influencing oligodendrocyte development. J. Neurochem. 64: 2396-2403 
14. Gavrieli Y, Sherman $Y$ and Benn-Sasson SA (1992) Identification of programmed cell death in situ via specific labeling of nuclear DNA fragmentation. J. Cell Biol. 119: 493-501

15. Cohen GM, Sun XM, Snowden RT, Dinsdale D and Skilletter DN (1992) Key morphological features of apoptotis may occur in the absence of internucleosomal DNA fragmentation. Biochem. J. 286: 331-334

16. Collins RJ, Harmon BV, Gabe GC and Kerr JFR (1992) Internucleosomal DNA cleavage should not be the sole criterion for identifying apoptosis. Int. J. Rad. Biol. 61: 451-453

17. Nakamura M, Yagi H, Kayaba S, Ishii T, Ohtsu S, Gotoh T and Itoh T (1995) Most thymocytes die in the absence of DNA fragmentation. Arch. Histol. Cytol. 58: $249-256$

18. Tomei LD, Shapiro JP and Cope FO (1993) Apoptosis in C3H/10T1/2 mouse embryonic cells: Evidence for internucleosomal DNA modification in the absence of double-strand cleavage. Proc. Natl. Acad. Sci. USA 90: 853-857

19. Zhang X, DeCarli AJ and Buttyan R (1997) Internucleosomal DNA fragmentation is not obligatory for castration induced rat ventral prostate cell apoptosis in vivo. Cell Death Differ. 4: 304-310

20. Collins J, SchandI CA, Young KK, Vesely Jand Willingham MC (1997) MajorDNA fragmentation is a late eventin apoptosis. J. Histochem. Cytochem. 45: 923-934

21. Enari M, Sakahira H, Yokoyama H, Okawa K, Iwamatsu A and Nagata S (1998) A caspase-activated DNase that degrades DNA during apoptosis, and its inhibitor ICAD. Nature 391: 43-50

22. Sakahira H, Enari M and Nagata $S$ (1998) Cleavage of CAD inhibitor in $C A D$ activation and DNA degradation during apoptosis. Nature 391: $96-99$

23. Duncan ID, Hammang JP, Goda S and Quarles RH (1989) Myelination in the jimpy mouse in the absence of proteolipid protein. Glia 2: 148-154
24. Williams II WC and Gard AL (1997) In vitro death of jimpy oligodendrocytes: Correlation with onset of DM-20/PLP expression and resistance to oligodendrogliotrophic factors. J. Neurosci. Res. 50: 177-189

25. Amur-Umarjee S, Phan T and Campagnoni AT (1993) Myelin basic protein mRNA translocation in oligodendrocytes is inhibited by astrocytes in vitro. J. Neurosci. Res. 36: $99-110$

26. Lachapelle F, Lapie P, Campagnoni AT and Gumpel M (1991) Oligodendrocytes of the jimpy phenotype can be partially restored by environmental factors in vivo. J. Neurosci. Res. 29: 235-243

27. Boison D and Stoffel W (1994) Disruption of the compacted myelin sheath of axons of the central nervous system in proteolipid protein-deficient mice. Proc. Natl. Acad. Sci. USA 91: 11709-11713

28. Klugmann M, Schwab MH, Puhlhofer A, Schneider A, Zimmermann F, Griffiths IR and Nave K-A (1997) Assembly of CNS myelin in the absence of proteolipid protein. Neuron 18: $59-70$

29. Knapp PE, Bartlett WP and Skoff RP (1987) Cultured oligodendrocytes mimic in vivo characteristics: Cell shape, expression of myelin specific antigens and membrane production. Dev. Biol. 120: 356-365

30. Sommer I and Schachner M (1982) Cells that are 04 antigen-positive and 01 antigen-negative differentiate into 01 antigen-positive oligodendrocytes. Neurosci. Lett. 29: 183-188

31. Bansal R, Warrington A, Gard A, Ranscht B and Pfeiffer S (1989) Multiple and novel specificities of monoclonal antibodies 01,04 and $\mathrm{R}-\mathrm{mAb}$ used in the analysis of oligodendrocyte development. J. Neurosci. Res. 24: 548-557

32. Knapp PE (1992) The cell cycle of glial cells grown in vitro: An immunocytochemical method of analysis. J. Histochem. Cytochem. 40: $1405-1411$ 